\title{
Revisión del sistema de vigilancia de lesiones y enfermedades durante juegos multideportivos \\ Review of the injury and illnesses surveillance system during multi-sport games
}

*Jeel Moya-Salazar, **Hugo Rodriguez-Papini, **Alejandro Opazo-Zamora, ***Vanessa Pineda-Vidangos, ****Victor Carpio-Quintana, *****Hans Contreras-Pulache

*Hospital Nacional Docente Madre Niño San Bartolomé (Perú), **Organización Deportiva Panamericana, (Estados Unidos), ***Proyecto Especial Legado Juegos Panamericanos y Parapanamericanos (Perú), *****Universidad Norbert Wiener (Perú)

Resumen. El objetivo de este estudio fuer presentar al Sistema deVigilancia de Lesiones y Enfermedades (SVLE) del Comité Olímpico Internacional (COI) diseñado para eventos multideportivos como un insumo para la planificación de los recursos necesarios para competencias deportivas. Desarrollamos una revisión sistemática siguiendo la guía PRISMA considerando como criterio de inclusión los eventos multideportivos con implementación de la SVLE del COI. La búsqueda fue realizada en los principales buscadores científicos (PubMed, Scopus, Scielo, ScientDirect, LILACS, y Latindex), en servidores públicos de pre-publicaciones (bioRxiv, SocArXiv, medRxiv y Preprints) y en metabuscadores (Google Scholar y Yahoo!). En la selección inicial se obtuvieron 367 estudios, incluyéndose 19 estudios para su análisis, donde solo 4 fueron deportes unitarios como fútbol, atletismo y balonmano. El SVLE del COI se ha usado inicialmente a gran escala en los Juegos Olímpicos de Beijing 2008 en 7 idiomas, al día de hoy más de 56,063 atletas en 19 eventos deportivos. En Sudamérica este sistema fue empleado en el I Juegos Deportivos Nacionales de Chile, los Juegos Olímpicos de Verano y los Juegos Olímpicos Rio 2016, y en los Juegos Panamericanos Lima 2019. Esta revisión muestra la experiencia documentada del SVLE del COI a lo largo de más de una década de uso de este instrumento, demostrando que el SVLE representa una herramienta útil, sencilla y ágil para el monitoreo de incidencias sanitarias.

Palabras clave: Traumatismos en Atleta, Vigilancia Sanitaria, Deportes, Atletas de élite, Juegos olímpicos, Medicina deportiva, Enfermedad.

\begin{abstract}
The objective of this study was to present the Injury and Illness Surveillance System (SVLE) of the International Olympic Committee (IOC) designed for multi-sport events as an input for planning the necessary resources for sports competitions. We developed a systematic review following the PRISMA guide, considering multi-sport events with implementation of the IOC SVLE as inclusion criteria. The search was carried out in the main scientific search engines (PubMed, Scopus, Scielo, ScientDirect, LILACS, and Latindex), in public pre-publication servers (bioRxiv, SocArXiv, medRxiv, and Preprints), and metasearch engines (Google Scholar and Yahoo!). In the initial selection, 367 studies were obtained, including 19 studies for analysis, where only 4 were unitary sports such as soccer, athletics, and handball. The IOC SVLE has initially been used on a large scale at the 2008 Beijing Olympic Games in 7 languages, monitoring today more than 56,063 athletes in 19 sporting events. In South America, this system was used in the I National Sports Games of Chile, the Summer Olympic Games and the Rio 2016 Olympic Games, and the Lima 2019 Pan American Games. This review shows the documented experience of the IOC SVLE throughout more of a decade of use of this instrument, demonstrating that the SVLE represents a useful, simple, and agile tool for monitoring health incidents.
\end{abstract}

Key words: Athletic Injuries, Health Surveillance, Sports, Elite athletes, Olympics, Sport medicine, illness.

\section{Introducción}

La historia de las competencias multideportivas data ya de varios cientos, sino miles, de años. Su desarrollo, desde la antigua Grecia hasta la actualidad, evidencia algunos rasgos constantes y preservados en historia de

Fecha recepción: 03-05-21. Fecha de aceptación: 30-09-21

Hans Contreras-Pulache

hans.contreras@uwiener.edu.pe la sociedad como el cultivo de una autodisciplina metódica, un ejercitar continuo y la exigencia propia asociada a todo entrenamiento de alto rendimiento o de competencia (Crowther, 2007). Ya en el mero disfrute por entretenimiento, o en el exhibicionismo artístico, incluso hasta en la competitividad tendente a la acción, los juegos multideportivos se constituyen, tradicional y culturalmente hablando, en eventos históricos que muestran al ser humano en los límites del trabajo riguroso sobre sí mismo (Crego, 2003). 
Así, en 1936, se desarrollaron los Juegos Olímpicos de Berlín, siendo el primer evento con una organización compleja y que utilizó el deporte para la difusión de ciertas ideologías (Joric, 2016). En efecto, las olimpiadas previstas para 1940 no pudieron llevarse a cabo a raíz de la Segunda Guerra Mundial. En la segunda mitad del siglo XX, los medios de comunicación magnificaron la difusión de las competencias multideportivas, convirtiéndolas en eventos a escala global de amplia aceptación y valoración de integración, bienestar y desarrollo de las capacidades humanas.

Según Sanz (2000), los recursos humanos de los eventos deportivos pueden ser de cinco tipos: ejecutivos, personal de servicio, personal técnico, voluntarios y de seguridad. Dentro del personal técnico se encuentran los especialistas médicos y profesionales deportivos que deben encargarse del registro concreto de las morbilidades suscitadas tanto en la competencia como en el entrenamiento. Esta tarea, a lo largo de los años, se ha ceñido a diversos sistemas de monitoreo de incidencias de morbilidades y sus registros (tanto para eventos individuales como para eventos multideportivos) que se han ido modificando a fin de obtener una tasa de respuesta y cobertura cada vez más fiel a la problemática vivida en el evento.

Los sistemas de vigilancia de lesiones de la Asociación Nacional de Atletas Colegiados (NCAA), de la Liga Nacional de Fútbol Americano (NFL), de la Federación Internacional de Fútbol Asociado (FIFA), de la Federación Internacional de Esquí (FIS), y de la Asociación Internacional de las Federaciones de Atletismo (IAAF) son alguno ejemplos usados en competiciones deportivas que han permitido profundizar en la recolección de datos sobre lesiones con el fin de analizarlos para la creación de intervenciones que permitan su prevención.

La presente revisión tiene como objetivo revisar la utilización del Sistema de Vigilancia de Lesiones y Enfermedades (SVLE) del Comité Olímpico Internacional (COI), sistema específicamente diseñado para eventos multideportivos, que permite un seguimiento de las incidencias sanitarias, y se constituye en herramienta de planificación para una gerencia basada en la evidencia.

\section{Método}

Desarrollamos una revisión sistemática siguiendo las recomendaciones de la guía PRISMA (Preferred Reporting Items for Systematic Reviews and MetaAnalyses) (Moher et al., 2009). Consideramos elegi- bles las publicaciones que han dado cuenta de la implementación del SVLE en distintos eventos deportivos. Incluimos estudios originales (prospectivos o retrospectivos), estudios de casos y controles, cartas científicas, que aborden eventos multideportivos y de atletas en particular, publicados en inglés, francés, portugués y español. Consideramos como criterios de exclusión los artículos de reflexión, revisiones sistemáticas, metaanálisis, y cartas editoriales (correspondencia).

Realizamos una búsqueda sistemática en los principales buscadores científicos (PubMed, Scopus, Scielo, ScientDirect, Web of Science, LILACS, y Latindex), en servidores públicos de pre-publicaciones (bioRxiv, SocArXiv, medRxiv y Preprints) y en metabuscadores (Google Scholar yYahoo!). Las publicaciones fueron identificadas usando los términos (((sistema de vigilancia)) AND ((lesiones deportivas) OR (enfermedades)) AND ((juego multideportivo) OR (evento deportivo)) AND (Comité Olímpico Internacional)) y las traducciones correspondientes para el inglés, francés y portugués. La búsqueda manual fue realizada entre el 15 y el 20 de febrero de 2021 y la revisión fue realizada independientemente por dos autores para verificar los criterios de inclusión de los estudios según PRISMA (Figura 1).

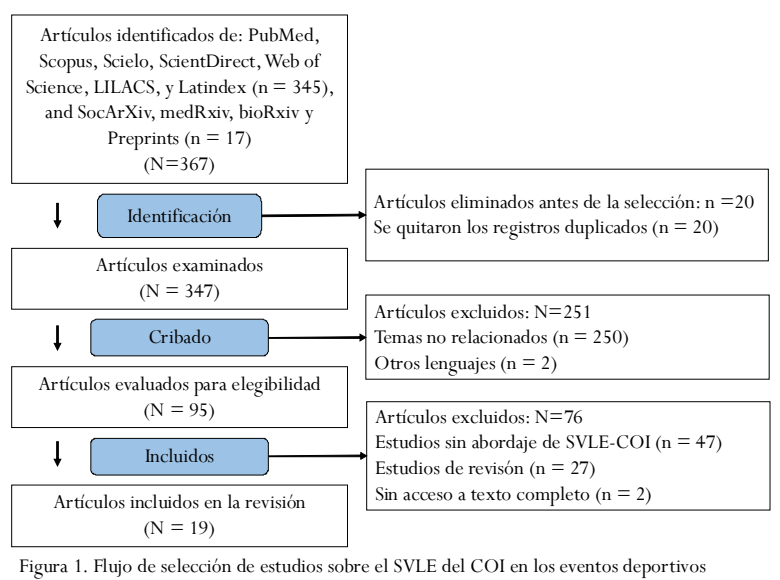

La extracción de datos fue supervisada por dos autores independientes, obteniéndose los datos del objetivo del estudio para su posterior análisis descriptivo con el uso de frecuencias y detalles de cada estudio (evento deportivo, país, total de atletas participantes, años de estudio, etc.). Esta revisión sistemática fue registrada en PROSPERO (ID282359) cumpliendo con los requisitos internacionales en buenas prácticas en investigación y publicaciones de revisiones sistemáticas.

\section{Resultados}

En la selección de estudios se obtuvo en la búsqueda 
inicial 367 estudios, en PubMed, Scopus, Scielo, ScientDirect, Web of Science, LILACS, y Latindex. En otros buscadores (SocArXiv, medRxiv, bioRxiv y Preprints) se identificaron 57. Se incluyeron 19 estudios para su análisis, donde solo 4 fueron desarrollados en eventos específicos para deportes unitarios como fútbol (2/19) (Dvorak et al., 2011; Muaidi, 2019), atletismo (Alonso et al., 2009), y balonmano (1/19) (Bere et al., 2015).

El SVLE del COI fue aplicado para más de 56,063 atletas en 19 eventos deportivos regionales (Engebretsen et al., 2010; Al-Shaqsi et al. 2012; Ruedl et al., 2012; Edouard et al., 2012; Edouard, Depiesse, Branco \& Alonso, 2014; Bere et al., 2015; Muaidi, 2019) y mundiales (Junge et al, 2009; Alonso et al., 2009; Mountjoy et al., 2010; Dvorak et al., 2011; Alonso et al., 2012; Soligard et al., 2015; Alonso et al., 2017; Soligard et al., 2019) permitiendo el registro de incidentes sanitarios (lesiones y enfermedades) durante los mismos. En Latinoamérica este sistema fue empleado en el I Juegos Deportivos Nacionales de Chile (Letelier \& Orizola, 2015), los Juegos Olímpicos de Verano 2016 en Rio de Janeiro (Soligard et al., 2017), Juegos Olímpicos Rio 2016 (Yoon et al., 2018), y, últimamente, en los Juegos Panamericanos Lima 2019 (Opazo-García et al., 2021).

El SVLE del COI se empezó a probar en las

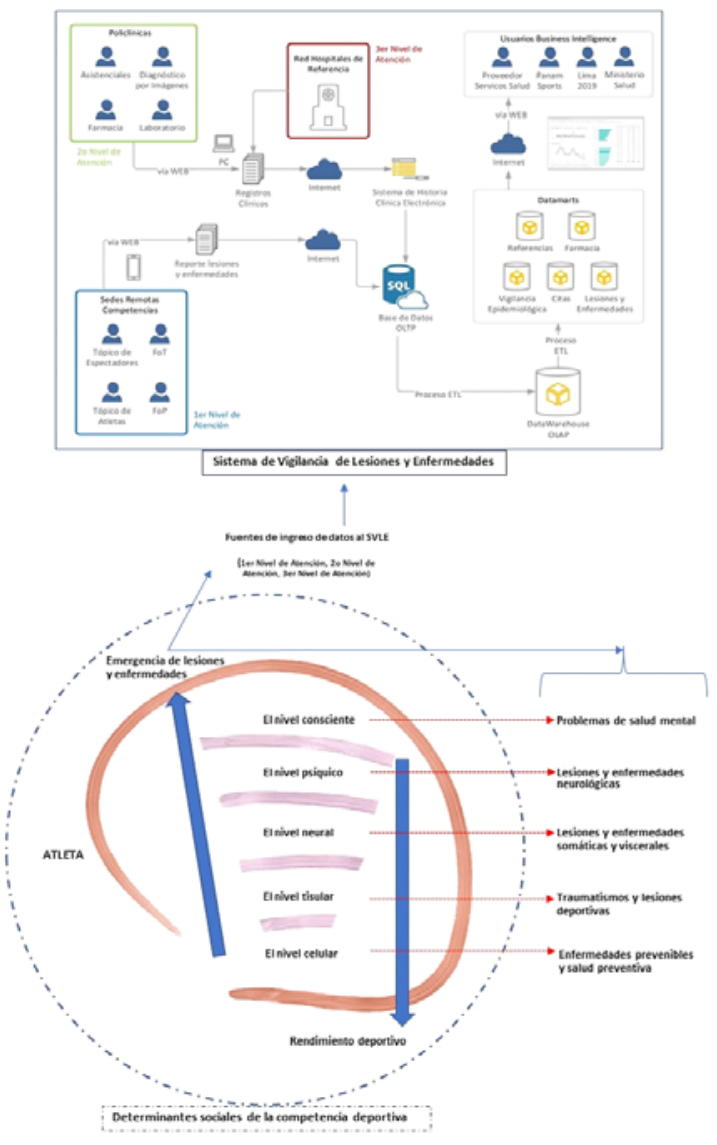

Figura 2. Modelo conceptual del atleta como sujeto de medición
Olimpiadas del 2004 (Junge et al., 2006) y en los Campeonatos Mundiales de la Asociación Internacional de Federaciones de Atletismo (IAAF) en el 2007 (Alonso et al., 2009), se puso en práctica a gran escala en los Juegos Olímpicos de Beijing 2008 (Junge et al., 2009). En este último evento, el registro de datos se apoyó en un formulario de reporte de lesiones disponible en siete idiomas (inglés, francés, chino, español, alemán, ruso y árabe) y logró una buena aceptación por parte de los distintos equipos médicos de las diferentes delegaciones deportivas, demostrando su facilidad y beneficios de aplicación en eventos multideportivos de gran tamaño. Sin embargo, Beijing dejó algunos asuntos pendientes, resueltos posteriormente, como por ejemplo la necesidad de contar con un seguimiento de los atletas lesionados (orientado a mejorar la precisión de los datos) y la inclusión de las lesiones y enfermedades crónicas por parte de los atletas (Junge et al., 2009). Dos años más tarde, una versión mejorada del SVLE se usó en los Juegos Olímpicos deVancouver 2010 (Engebretsen et al., 2010).

Actualmente (Tabla 1) existen 15 diferentes sistemas de vigilancia de lesiones y enfermedades vigentes (Ekegren, Gabbe \& Finch, 2015). De estos, 6 (40\%) están destinados a eventos de fútbol (o relacionados a este tipo de deporte), otros $6(40 \%)$ son aplicables a eventos multideportivos (1 béisbol, 1 esquí y snowboard, 1 cricket). Cinco de estos sistemas 5 (33.3\%) fueron originados en Europa, 6 (40\%) en los Estados Unidos y 2 (13.3\%) en Australia. No obstante, los 2 (13.3\%) restantes no poseen una afiliación nacional única (Ekegren, Gabbe \& Finch 2015).

Estos 15 sistemas han estado activos por un periodo que oscila entre 5 y 34 años, siendo el de la Liga Nacional de Fútbol el más antiguo (1980 hasta ahora). La última aplicación del SVLE ha sido durante los Juegos Panamericanos Lima 2019, donde se ha demostrado emergencias dentales en $1.14 \%$ de atletas, siendo las más frecuentes las enfermedades peri-odontológicas (34\%) y caries dental (29\%) principalmente en atletas de alto rendimiento y futbolistas (Opazo-García et al., 2021).

El 46.4\% (7/15 sistemas) emplean definiciones de lesiones relacionadas con la pérdida de tiempo debidas a la participación en el deporte, otras 3 (20\%) usan definiciones de lesiones de atención médica (donde una lesión reportable debe requerir algún tipo de atención médica), 2 (13.3\%) sistemas utilizan definiciones que incluyen ambos: pérdida de tiempo y atención médica. Por otro lado, en 8 (53.3\%) sistemas los médicos del 
Tabla 1.

Sistemas de Vigilancia de lesiones implementados entre 1982 y 2021 (*) $^{2}$

\begin{tabular}{|c|c|c|c|c|c|c|c|}
\hline Nombre del sistema & $\begin{array}{l}\text { Organización que propuso el } \\
\text { sistema }\end{array}$ & $\begin{array}{l}\text { Año de } \\
\text { publicación }\end{array}$ & $\begin{array}{l}\text { Deportes } \\
\text { considerados }\end{array}$ & País de origen & Población evaluada & ¿Quién recolecta la información? & $\begin{array}{l}\text { ¿Cómo se recolectan los } \\
\text { datos? }\end{array}$ \\
\hline $\begin{array}{l}\text { Sistema de vigilancia de } \\
\text { lesiones de la Liga Nacional } \\
\text { de Fútbol Americano (NFL) }\end{array}$ & NFL & $\begin{array}{l}1998 \text { en } \\
\text { adelante }\end{array}$ & $\begin{array}{l}\text { Fútbol americano } \\
\text { profesional }\end{array}$ & USA & $\begin{array}{l}\text { Masculino. Jugadores } \\
\text { profesionales de } \\
\text { fútbol americano }\end{array}$ & Entrenadores deportivos certificados & $\begin{array}{l}\text { Sistema de vigilancia en } \\
\text { línea }\end{array}$ \\
\hline $\begin{array}{l}\text { Sistema de vigilancia de } \\
\text { lesiones de la Asociación } \\
\text { Nacional de Atletas } \\
\text { Colegiados (NCAA) }\end{array}$ & NCAA & $\begin{array}{l}1982 \text { en } \\
\text { adelante }\end{array}$ & Multideportivo & USA & $\begin{array}{l}\text { Atletas universitarios } \\
\text { masculinos y } \\
\text { femeninos. }\end{array}$ & Entrenadores deportivos certificados & $\begin{array}{l}\text { Sistema de vigilancia en } \\
\text { línea }\end{array}$ \\
\hline $\begin{array}{l}\text { Encuesta anual de lesiones } \\
\text { de la Liga Australiana de } \\
\text { Fútbol (AFL) }\end{array}$ & AFL & $\begin{array}{l}1992 \text { en } \\
\text { adelante (todos } \\
\text { los jugadores } \\
\text { desde 1996) } \\
\end{array}$ & Fútbol australiano & Australia & $\begin{array}{l}\text { Masculino. Jugadores } \\
\text { profesionales de } \\
\text { fútbol australiano }\end{array}$ & Equipo de personal médico & $\begin{array}{l}\text { Registros electrónicos } \\
\text { presentados al } \\
\text { coordinador del estudio al } \\
\text { final de la temporada } \\
\end{array}$ \\
\hline $\begin{array}{l}\text { Base de datos del sistema de } \\
\text { vigilancia de lesiones de la } \\
\text { escuela pública del condado } \\
\text { de Fairfax }\end{array}$ & $\begin{array}{l}\text { Sistema de la escuela pública } \\
\text { del condado de Fairfax }\end{array}$ & $\begin{array}{l}1997 \text { en } \\
\text { adelante }\end{array}$ & $\begin{array}{l}27 \text { deportes de la } \\
\text { escuela } \\
\text { secundaria. }\end{array}$ & USA & $\begin{array}{l}\text { Atletas masculinos y } \\
\text { femeninos de la } \\
\text { escuela secundaria. }\end{array}$ & Entrenadores deportivos certificados & $\begin{array}{l}\text { Sistema de registro } \\
\text { médico electrónico } \\
\text { enviado al coordinador de } \\
\text { investigación presente. }\end{array}$ \\
\hline $\begin{array}{l}\text { Sistema de vigilancia de la } \\
\text { Federación Internacional de } \\
\text { Fútbol Asociado (FIFA) } \\
\text { (Enforque COI para } \\
\text { deportes de equipo) }\end{array}$ & FIFA & $\begin{array}{l}1998 \text { en } \\
\text { adelante }\end{array}$ & Fútbol & Suiza & $\begin{array}{l}\text { Masculino y } \\
\text { femenino. Jugadores } \\
\text { profesionales de } \\
\text { fútbol que } \\
\text { participaron en todas } \\
\text { las competiciones de } \\
\text { la FIFA }\end{array}$ & Equipo de médicos. & $\begin{array}{l}\text { Formularios en papel } \\
\text { completados después de } \\
\text { cada partido y recogidos } \\
\text { por un representante de la } \\
\text { FIFA }\end{array}$ \\
\hline $\begin{array}{l}\text { Encuesta de lesiones de } \\
\text { cricket en Australia }\end{array}$ & Cricket Australia & $\begin{array}{l}1998 \text { en } \\
\text { adelante }\end{array}$ & Cricket & Australia & $\begin{array}{l}\text { Masculino. Jugadores } \\
\text { de cricket } \\
\text { profesionales }\end{array}$ & Médico o fisioterapeuta del equipo & $\begin{array}{l}\text { Base de datos electrónica } \\
\text { de lesiones }\end{array}$ \\
\hline $\begin{array}{l}\text { Estudio de lesiones de la liga } \\
\text { de campeones de las } \\
\text { Asociaciones Europeas de } \\
\text { Fútbol (UEFA) }\end{array}$ & UEFA & $\begin{array}{l}2001 \text { en } \\
\text { adelante }\end{array}$ & Fútbol & $\begin{array}{l}\text { Múltiples } \\
\text { países } \\
\text { europeos }\end{array}$ & $\begin{array}{l}\text { Jugadores de fútbol } \\
\text { profesionales } \\
\text { masculinos de los } \\
\text { clubes de fútbol } \\
\text { europeos de primera } \\
\text { división } \\
\end{array}$ & Equipo de personal médico & $\begin{array}{l}\text { Sistema de vigilancia en } \\
\text { línea }\end{array}$ \\
\hline $\begin{array}{l}\text { Liga noruega de fútbol } \\
\text { profesional }\end{array}$ & $\begin{array}{l}\text { Liga profesional masculina } \\
\text { noruega (Tipperligaen) }\end{array}$ & $\begin{array}{l}2000 \text { en } \\
\text { adelante }\end{array}$ & fútbol & Noruega & $\begin{array}{l}\text { Masculino, jugadores } \\
\text { de fútbol } \\
\text { profesionales }\end{array}$ & Médico o fisioterapeuta del equipo & $\begin{array}{l}\text { Formularios en papel } \\
\text { recopilados } \\
\text { mensualmente. }\end{array}$ \\
\hline $\begin{array}{l}\text { Proyecto de vigilancia de } \\
\text { lesiones de rugby profesional } \\
\text { en Inglaterra }\end{array}$ & $\begin{array}{l}\text { La Unión Futbol- Rugby } \\
\text { (RFU) y la Premier Rugby Ltd } \\
\text { (PRL) }\end{array}$ & $\begin{array}{l}2002 \text { en } \\
\text { adelante }\end{array}$ & Unión de Rugby & Inglaterra & $\begin{array}{l}\text { Masculino. Jugadores } \\
\text { profesionales de la } \\
\text { unión de rugby }\end{array}$ & $\begin{array}{l}\text { Personal médico de cada club de Premiers e } \\
\text { realizó una revisión estructurada de la } \\
\text { literatura a fin de identificar las publicaciones } \\
\text { más relevantes sobre los sistemas de vigilancia } \\
\text { de lesiones deportivas. Esta búsqueda } \\
\text { sistemática se fundamentó en las siguientes } \\
\text { bases de datos: Scopus (todos los años), } \\
\text { MEDLINE (1946 en adelante), PubMed } \\
\text { (todos los años), Embase (1947 en adelante), } \\
\text { Google Scholar (todos los años), CINAHL } \\
\text { plus (1982 en adelante) y ScienceDirect } \\
\text { (todos los años). Las palabras claves para dicha } \\
\text { estrategia de búsqueda fueron: Sports Injury } \\
\text { and Illness Incidence, Injury and Illness, } \\
\text { Olympic Games, Surveillance System. El } \\
\text { personal de fuerza y acondicionamiento } \\
\text { registra los horarios semanales de } \\
\text { entrenamiento y exposición }\end{array}$ & $\begin{array}{l}\text { Base de datos electrónica } \\
\text { de lesiones. }\end{array}$ \\
\hline $\begin{array}{l}\text { Sistema de vigilancia de } \\
\text { lesiones relacionadas al } \\
\text { deporte en la escuela } \\
\text { secundaria nacional. }\end{array}$ & $\begin{array}{l}\text { Centro de investigación de } \\
\text { lesiones y política en el hospital } \\
\text { infantil de Columbus y el } \\
\text { Programa de Prevención de } \\
\text { Lesiones, Educación e } \\
\text { Investigación (PIPER) en la } \\
\text { Escuela de Salud Pública de } \\
\text { Colorado, Universidad de } \\
\text { Colorado. }\end{array}$ & $\begin{array}{l}2005 \text { en } \\
\text { adelante }\end{array}$ & Multideportivo & USA & $\begin{array}{l}\text { Atletas masculinos y } \\
\text { femeninos de la } \\
\text { escuela secundaria. }\end{array}$ & Entrenadores de atletismo certificados & $\begin{array}{l}\text { Sistema de vigilancia de } \\
\text { lesiones en línea }\end{array}$ \\
\hline $\begin{array}{l}\text { Sistema de vigilancia de } \\
\text { lesiones de la Federación } \\
\text { Internacional de Esquí (FIS) }\end{array}$ & $\begin{array}{l}\text { FIS y el Centro de } \\
\text { Investigación de Traumatismos } \\
\text { Deportivos de Oslo (OSTRC) }\end{array}$ & $\begin{array}{l}2006 \text { en } \\
\text { adelante }\end{array}$ & $\begin{array}{l}\text { Esquí de élite y } \\
\text { tabla sobre nieve } \\
\text { (snowboarding) }\end{array}$ & Noruega & $\begin{array}{l}\text { Masculino y } \\
\text { femenino. } \\
\text { Esquiadores y } \\
\text { practicantes de } \\
\text { snowboard de la } \\
\text { Copa del Mundo. }\end{array}$ & $\begin{array}{l}\text { (1) Delegados técnicos (TDs) con asistencia } \\
\text { de personal médico, (2) entrevista a atletas/ } \\
\text { entrenadores. }\end{array}$ & $\begin{array}{l}\text { Formularios en papel o } \\
\text { electrónicos completados } \\
\text { por los TDs } \\
\text { posteriormente enviados } \\
\text { vía fax o mail regular a la } \\
\text { oficina del FIS, (2)los } \\
\text { atletas (o sus } \\
\text { entrenadores/ personal } \\
\text { médico) entrevistados por } \\
\text { los investigadores al final } \\
\text { de la temporada. }\end{array}$ \\
\hline $\begin{array}{l}\text { Sistema de vigilancia de la } \\
\text { Asociación Internacional de } \\
\text { las Federaciones de } \\
\text { Atletismo (IAAF) (enfoque } \\
\text { IOC para deportes } \\
\text { individuales) }\end{array}$ & IAAF & $\begin{array}{l}2007 \text { en } \\
\text { adelante }\end{array}$ & Atletismo & $\begin{array}{l}\text { Múltiples } \\
\text { países }\end{array}$ & $\begin{array}{l}\text { Atletas de élite } \\
\text { masculinos y } \\
\text { femeninos en } \\
\text { deportes individuales. }\end{array}$ & Médicos o fisioterapeutas del equipo & $\begin{array}{l}\text { Formularios en papel } \\
\text { completados diariamente } \\
\text { durante los campeonatos. }\end{array}$ \\
\hline $\begin{array}{l}\text { Sistema de vigilancia de } \\
\text { lesiones para eventos } \\
\text { multideportivos del Comité } \\
\text { Olímpico Internacional } \\
\text { (IOC) }\end{array}$ & IOC & $\begin{array}{l}2008 \text { en } \\
\text { adelante }\end{array}$ & Multideportivo & $\begin{array}{l}\text { Múltiples } \\
\text { países }\end{array}$ & $\begin{array}{l}\text { Atletas Olímpicos } \\
\text { masculinos y } \\
\text { femeninos. }\end{array}$ & $\begin{array}{l}\text { Personal médico calificado (médico o } \\
\text { fisioterapeuta del equipo) }\end{array}$ & $\begin{array}{l}\text { Formularios en papel o } \\
\text { electrónicos disponibles } \\
\text { en ocho idiomas. }\end{array}$ \\
\hline $\begin{array}{l}\text { Red de Investigación Basada } \\
\text { en la Práctica de } \\
\text { Entrenamiento Atlético (AT- } \\
\text { PBRN) }\end{array}$ & $\begin{array}{l}\text { Todavía Universidad de } \\
\text { Atletismo }\end{array}$ & $\begin{array}{l}2009 \text { en } \\
\text { adelante }\end{array}$ & Multideportivo & USA & $\begin{array}{l}\text { Atletas masculinos y } \\
\text { femeninos de la } \\
\text { escuela secundaria. }\end{array}$ & Entrenadores de atletismo profesionales & $\begin{array}{l}\text { Sistema de vigilancia } \\
\text { online }\end{array}$ \\
\hline $\begin{array}{l}\text { Sistema de vigilancia de } \\
\text { lesiones de la Liga Mayor de } \\
\text { Baseball }\end{array}$ & Liga Mayor de Baseball & $\begin{array}{l}2010 \text { en } \\
\text { adelante }\end{array}$ & Baseball & USA & $\begin{array}{l}\text { Masculino. Jugadores } \\
\text { de baseball } \\
\text { profesionales }\end{array}$ & $\begin{array}{l}\text { Entrenadores de atletismo certificados y } \\
\text { médicos del equipo. }\end{array}$ & Historia clínica electrónica \\
\hline
\end{tabular}

$\left({ }^{*}\right)$ : En el marco de la crisis sanitaria global, durante el año 2020, y en lo que va del año 2021, no se han llevado a cabo eventos deportivos de competencia 
equipo o fisioterapeutas son los responsables de registrar los datos de lesiones; en cambio, para los 6 (40\%) sistemas originados en los Estados Unidos, son los entrenadores de atletismo certificados quienes registran los datos.

En un sistema, el personal técnico no capacitado médicamente es el encargado de completar los formularios de informes de lesiones, y lo complementa con entrevistas a los atletas o a los entrenadores y la asistencia del personal médico. Cinco (33.3\%) sistemas utilizan formularios en línea que permiten cargar los detalles de las lesiones directamente en una base de datos central, otros 5 (33.3\%) sistemas incorporan un formulario electrónico o una hoja de cálculo, 4 (26.7\%) utilizan formularios en papel y 2 (13.3\%) ofrecen la opción de utilizar formularios electrónicos o en papel (Ekegren, Gabbe \& Finch, 2015).

\section{Discusión}

Los sistemas de vigilancia de lesiones surgen de la necesidad de identificar, analizar y monitorear las constantes sanitarias inherentes a los eventos deportivos (de diversa naturaleza), a fin de que puedan sugerirse medidas preventivas e implementarse normativas que favorezcan la salud del atleta durante el entrenamiento o la competición. Entre los sistemas de vigilancia que se han propuesto hasta nuestros días, priman los destinados a eventos en disciplinas particulares, y solo en el curso de las últimas dos décadas se pueden ver esfuerzos por implementar sistemas de vigilancia aplicados a eventos multideportivos. Cabe destacar aquí, el estudio de los Juegos Olímpicos del 2004 (Junge et al., 2006) ya que, debido a la alta calidad de los datos obtenidos en ocho deportes, su cumplimiento excelente y su buena acogida por los miembros de los equipos médicos (Junge et al., 2009), logró cimentar las bases para el desarrollo del SVLE.

Una limitación del SVLE es la falta de dialogo con los sistemas de monitoreo sanitario en varios países. En el contexto de gobierno digital es necesario que las plataformas de manejo de atletas durante los eventos deportivos puedan acceder a la información de cada participante para entender el contexto de su salud previa a la competición y lograr mejorar la precisión de su atención. En el siguiente acápite vamos a detallar las especificaciones para el SVLE del COI desde el año 2008 hasta la actualidad.

\section{Sistema de Vigilancia de Lesiones y Enferme-}

\section{dades (SVLE) del Comité Olímpico Internacional} (COI) para eventos multideportivos

Se trata de un método estandarizado y diseñado específicamente para registrar las incidencias de lesiones y enfermedades suscitadas en los eventos multideportivos de alto rendimiento y, en base a ello, desprender estrategias preventivas en pro de la salud de los atletas. A lo largo de este siglo, el método ha demostrado tener una aceptación excelente, a la vez que permite una evaluación homogénea e integral de la población sujeta a evaluación (empezando por los atletas, pero pudiendo ampliarse a todo el personal que participa en el proceso organizativo de una competencia). Dada su naturaleza estandarizada, facilita la realización de comparaciones entre los estudios que emplean esta misma metodología. En la Tabla 2, se resume la experiencia acumulada de la aplicación del SVLE del COI hasta el momento en que se escriben estas líneas.

Este sistema se usó por primera vez en los Juegos Olímpicos de Beijing 2008, en una experiencia sistematizada y publicada por Junge et al., (2009). En ese entonces el sistema ya contaba con una definición de «lesión» consensuada y con normativas que le permitieron analizar las características, incidencias y etiologías de las lesiones de manera efectiva. Posteriormente, el sistema se optimizó incluyendo el registro de enfermedades (no solo lesiones) y se puso en marcha así en los Juegos Olímpicos de Invierno realizado en Vancouver 2010. Por otra parte, en competencias de un único deporte también ha demostrado ser viable y confiable en tanto sistema de recolección de datos (Letelier \& Orizola, 2013). Desde entonces, el SVLE del COI se ha implementado principalmente en Londres 2012 (Engebretsen et al., 2013), Sochi 2014 (Soligard et al., 2015), Río 2016 (Yoon, Bae, Kang \& Kim, 2018) y Pyeong Chang 2018 (Soligard et al., 2019). Su última aplicación fue en los Juegos Panamericanos Lima 2019 (Opazo-García et al., 2021).

Es preciso resaltar la importancia de ciertos requisitos que asegurarán el éxito de la implementación del SVLE y su posicionamiento a lo largo del desarrollo del evento. Primero, asegurar la adherencia de los miembros de los equipos médicos tanto de los diversos países como del país anfitrión. Segundo, es necesario garantizar un adecuado procedimiento de recolección de datos, en la medida de lo posible contando con lo digital como elemento transversal. Tercero, es casi obligatoria la realización de una reunión de instrucción (llevada a cabo días previos al evento deportivo) que garantice el conocimiento por todos los comités médicos de cada 
delegación y comprometa su participación; el SVLE cuenta con un folleto instructivo que detalla el protocolo del sistema, siendo estos documentos oficiales y provistos por el COI. Es en esta reunión que debe clarificarse un hecho trascendental: el SVLE guarda en estricta reserva el carácter confidencial de los datos de los atletas. Esto es de crucial importancia ya que, de no hacerlo, constituiría la primera limitante al momento de la recolección de datos debido a que muchos países no desean, entendiblemente, revelar la condición física y de salud de sus atletas. En este sentido, la confidencialidad de la información y el anonimato asegurado deberán presentarse, y resaltarse, como fortalezas del SVLE del COI. Otra ventaja importante radica en que los miembros del grupo del estudio se encuentran en constante contacto personal con los miembros de los equipos médicos de las delegaciones a fin de garantizar un cumplimiento óptimo y de asegurar el propósito común, un correcto y continuo proceso de levantamiento de información.

Adentrándonos en el reporte empleado en la recolección de datos, es vital describir de manera detallada sus puntos constituyentes. Para registrar las lesiones se requieren datos como: número de acreditación del atleta, deporte y prueba; en qué instancia de la competencia/entrenamiento se dio el hecho; fecha y hora de la lesión; parte del cuerpo lesionada; tipo y causa de la lesión; una estimación de la duración esperada de la ausencia de la competencia y/o entrenamiento. Para enfermedades, se requieren datos como: número de acreditación del atleta, deporte y prueba; diagnóstico; fecha de aparición de los síntomas; sistema afectado; síntomas principales; causa de enfermedad; y, una estimación de la duración esperada de la ausencia de la competencia y/o entrenamiento. En breves líneas, el SVLE del COI consiste en un método de evaluación estandarizado que

Tabla 2.

Experiencia acumulada del SVLE del COI 2008-2021(*)

\begin{tabular}{|c|c|c|c|c|c|}
\hline Autores & Evento deportivo & $\begin{array}{l}\text { Deportes } \\
\text { considerados }\end{array}$ & Países involucrados & Total de deportistas & Resultado principal \\
\hline Junge A, et al. & $\begin{array}{l}\text { Juegos Olímpicos de Verano } \\
2008\end{array}$ & Multideportivo & Múltiples países & 10977 atletas & $\begin{array}{l}1055 \text { lesiones reportadas. } \\
\text { Diagnóstico de lesión más frecuente: esguinces de tobillo y desgarros. } \\
\text { Mayor incidencia de lesiones: fútbol, taekwondo, hockey, balonmano, levantamiento de } \\
\text { pesas y boxeo. }\end{array}$ \\
\hline Alonso JM, Junge A & $\begin{array}{l}\text { Campeonatos mundiales de } \\
\text { atletismo de la IAAF } 2007\end{array}$ & Atletismo & 200 naciones & 1980 atletas & $\begin{array}{l}192 \text { lesiones reportadas. } \\
\text { Diagnóstico de lesión más frecuente: distensión muscular. } \\
\text { Mayor incidencia de lesiones: maratón, heptatlón, } 800 \mathrm{~m}, 10,000 \mathrm{~m} \text {. }\end{array}$ \\
\hline $\begin{array}{l}\text { Engebretsen L, } \\
\text { Steffen K, et al. }\end{array}$ & $\begin{array}{l}\text { XXI Juegos olímpicos de } \\
\text { invierno 2010, Vancouver }\end{array}$ & Multideportivo & 82 naciones & 2567 atletas & $\begin{array}{l}287 \text { lesiones y } 185 \text { enfermedades reportadas. } \\
\text { Mayor incidencia de lesiones: snowboard cross, bobsleigh, hockey sobre hielo, pista } \\
\text { corta y estilo libre alpino. } \\
\text { Lugar de lesión más común: cara, cabeza, columna cervical y rodilla. }\end{array}$ \\
\hline $\begin{array}{l}\text { Mountjoy M, Junge } \\
\text { A, et al. }\end{array}$ & $\begin{array}{l}\text { Campeonato Mundial FINA } \\
2009 \text { (acuáticos) }\end{array}$ & Multideportivo & 172 países & 2592 atletas & $\begin{array}{l}171 \text { lesiones y } 184 \text { enfermedades reportadas. Lugar de lesión más común: hombro, } \\
\text { cabeza. Mayor incidencia de lesiones: buceo. }\end{array}$ \\
\hline Letelier A, Orizola A & $\begin{array}{l}\text { I Juegos Deportivos } \\
\text { Nacionales de Chile }\end{array}$ & Multideportivo & 15 regiones & 1229 atletas & $\begin{array}{l}66 \text { lesiones y } 10 \text { enfermedades reportadas. } \\
\text { Mayor incidencia de lesiones: taekwondo y gimnasia rítmica. }\end{array}$ \\
\hline $\begin{array}{l}\text { Engebretsen, } \\
\text { SoligardT, et al. }\end{array}$ & $\begin{array}{l}\text { Juegos Olímpicos de Verano } \\
\text { 2012, Londres }\end{array}$ & Multideportivo & 204 países & 10568 atletas & $\begin{array}{l}1361 \text { lesiones y } 758 \text { enfermedades reportadas. Mayor incidencia de lesiones: atletismo y } \\
\text { fútbol. } \\
\text { Mayor incidencia de enfermedades: atletismo y natación. }\end{array}$ \\
\hline $\begin{array}{l}\text { Soligard T, Steffen K, } \\
\text { et al. }\end{array}$ & $\begin{array}{l}\text { Juegos Olímpicos de Verano } \\
\text { 2016, Rio de Janeiro }\end{array}$ & Multideportivo & 207 países & 11274 atletas & $\begin{array}{l}1101 \text { lesiones y } 651 \text { enfermedades reportadas. Mayor incidencia de lesiones: ciclismo } \\
\text { BMX. } \\
\text { Mayor incidencia de enfermedades: buceo, maratón en aguas abiertas y vela. }\end{array}$ \\
\hline Soligard T, et al. & $\begin{array}{l}\text { Juegos Olímpicos de } \\
\text { Invierno 2014, Sochi. }\end{array}$ & Multideportivo & 88 países & 2780 atletas & $\begin{array}{l}391 \text { lesiones y } 249 \text { enfermedades reportadas. Mayor incidencia de lesiones: esquí aéreo } \\
\text { Mayor incidencia de enfermedades: trineo simple (skeleton). }\end{array}$ \\
\hline $\begin{array}{l}\text { Dvorak J, Junge A, } \\
\text { Derman W, et al. }\end{array}$ & Copa Mundial FIFA 2010 & fútbol & 26 países & 736 jugadore & $\begin{array}{l}\text { s } 229 \text { lesiones (del total) y } 99 \text { enfermedades (de } 89 \text { jugadores) reportadas. Diagnóstico de } \\
\text { lesión más frecuente: tensión muscular y esguince de tobillo. }\end{array}$ \\
\hline Alonso JM, et al. & $\begin{array}{l}\text { Campeonato mundial de } \\
\text { Atletismo 2011, Daegu }\end{array}$ & Multideportivo & 201 países & 1851 atletas & $\begin{array}{l}249 \text { lesiones y } 126 \text { enfermedades reportadas. } \\
\text { Diagnóstico de lesión más frecuente: distensión de los isquiotibiales. } \\
\text { Diagnóstico de enfermedad más frecuente: infección del tracto respiratorio superior. }\end{array}$ \\
\hline $\begin{array}{l}\text { Ruedl G, } \\
\text { Schobersberg erW, } \\
\text { et al. }\end{array}$ & $\begin{array}{l}\text { Juegos Olímpicos de la } \\
\text { Juventud de Invierno 2012, } \\
\text { Innsbruck, Austria }\end{array}$ & Multideportivo & 69 naciones & 1021 atletas & $\begin{array}{l}111 \text { lesiones y } 86 \text { enfermedades reportadas. Mayor incidencia de lesiones: esquí halfpipe } \\
\text { Lugar de lesión más común: rodilla. }\end{array}$ \\
\hline $\begin{array}{l}\text { Alonso JM, Tscholl } \\
\text { PM, et al. }\end{array}$ & $\begin{array}{l}\text { Campeonatos Mundiales de } \\
\quad \text { Atletismo } \\
\text { 2009, Berlín }\end{array}$ & Multideportivo & 200 países & 1979 atletas & $\begin{array}{l}236 \text { lesiones y } 135 \text { enfermedades reportadas. } \\
\text { Diagnóstico de lesión más frecuente: tensión en el muslo. } \\
\text { Diagnóstico de enfermedad más frecuente: infección del tracto respiratorio superior. }\end{array}$ \\
\hline $\begin{array}{l}\text { Bere T, JM Alonso, et } \\
\text { al. }\end{array}$ & $\begin{array}{l}\text { XXIV Campeonato Mundial } \\
\text { de Balonmano Masculino } \\
\text { 2015, Qatar. }\end{array}$ & Balonmano & 24 equipos & 384 atletas & $\begin{array}{l}132 \text { lesiones y } 42 \text { enfermedades reportadas. Lugar de lesión más común: tobillo. } \\
\text { Diagnóstico de lesión más frecuente: contusiones, esguinces o tensiones. }\end{array}$ \\
\hline $\begin{array}{l}\text { Edouard P, Depiesse } \\
\text { F, Branco P, Alonso } \\
\text { JM }\end{array}$ & $\begin{array}{l}\text { Campeonato Europeo de } \\
\text { Atletismo 2012, Helsinki. }\end{array}$ & Multideportivo & 50 países & 1244 atletas & $\begin{array}{l}133 \text { lesiones y } 27 \text { enfermedades reportadas. } \\
\text { Diagnóstico de lesión más frecuente: distensión de los isquiotibiales Diagnóstico de } \\
\text { enfermedad más frecuente: infección del tracto respiratorio superior. }\end{array}$ \\
\hline $\begin{array}{l}\text { Edouard P, Depiesse } \\
\text { F, Hertert P, Branco } \\
\text { P, Alonso JM. }\end{array}$ & $\begin{array}{l}\text { Campeonato Europeo de } \\
\text { Atletismo en Pista Cubierta } \\
\text { 2011, París }\end{array}$ & Multideportivo & 46 países & 631 atletas & $\begin{array}{l}20 \text { lesiones y } 18 \text { enfermedades reportadas. Diagnóstico de lesión más frecuente: } \\
\text { tensión muscular } \\
\text { Diagnóstico de enfermedad más frecuente: infección del tracto respiratorio superior }\end{array}$ \\
\hline $\begin{array}{l}\text { Al-Shaqsi S, Al- } \\
\text { Kashimiri A, Al-Risi } \\
\text { A, Al- Mawali S. }\end{array}$ & $\begin{array}{l}\text { - Segundos Juegos Asiáticos de } \\
\text { i Playa }\end{array}$ & Multideportivo & $\begin{array}{l}\text { Países miembros del } \\
\text { Consejo Olímpico de } \\
\text { Asia }\end{array}$ & 1132 atletas & $\begin{array}{l}177 \text { lesiones y } 118 \text { enfermedades reportadas. Lugar de lesión más común: pie / dedo del } \\
\text { pie. El sistema más afectado fue el tracto respiratorio. }\end{array}$ \\
\hline $\begin{array}{l}\text { Soligard T, Palmer D, } \\
\text { Steffen K }\end{array}$ & $\begin{array}{l}\text { Juegos Olímpicos de } \\
\text { Invierno 2018, } \\
\text { PyeongChang, }\end{array}$ & Multideportivo & 92 países & 2914 atletas & $\begin{array}{l}376 \text { lesiones y } 279 \text { enfermedades reportadas. Mayor incidencia de lesiones: esquí } \\
\text { halfpipe Mayor incidencia de enfermedades: biatlón } \\
\text { El } 70 \% \text { de las enfermedades afectaron el sistema respiratorio. }\end{array}$ \\
\hline Muaidi & $\begin{array}{l}\text { Liga Profesional Saudí } \\
\text { durante la temporada de } \\
\text { fútbol 2015-2016 }\end{array}$ & Fútbol & Arabia Saudita & 14 clubes de fútbol & $\begin{array}{l}386 \text { lesiones y } 65 \text { enfermedades reportadas. } \\
\text { Diagnóstico de lesión más frecuente: contusiones, hematomas y moretones. Diagnóstico } \\
\text { de enfermedad más frecuente: infecciones del tracto respiratorio y gastroenteritis. }\end{array}$ \\
\hline JYoon, et al. & Juegos Olímpicos 2016, Rio & Multideportivo & Corea & 204 atletas & $\begin{array}{l}308 \text { lesiones y } 73 \text { enfermedades reportadas } \\
\text { Mayor incidencia de lesiones: boxeo y la lucha libre, mientras que la tasa más alta de } \\
\text { enfermedades se observó en el boxeo y en natación. El } 75 \% \text { de todas las infecciones fue } \\
\text { del tracto respiratorio. }\end{array}$ \\
\hline
\end{tabular}


proporciona no solo información epidemiológica importante sobre el número, características y etiologías de los problemas de salud, sino que también establece instrucciones para la prevención de lesiones y permite monitorear cambios a largo plazo en la frecuencia y circunstancias de la lesión (Junge et al., 2009). Este sistema integrado brinda ventajas ya que no requiere un post-tratamiento de datos como se ha visto en otros sistemas (González-Ravé et al., 2017).

En el caso de la más reciente aplicación del SVLE que ha tenido lugar es en los Juegos Panamericanos y Parapanamericanos Lima 2019 (Opazo-García et al., 2021), el flujo de la información clínica se realizó de manera electrónica a través de un sistema de información para concluir en analítica de datos, en tiempo real, a fin de monitorear la gestión de las atenciones, monitoreo de tendencias, estado de pacientes y eventuales focos epidemiológicos. En el primer nivel de atención, situado en las sedes de competencias, los profesionales asistenciales registran sus atenciones de primeros auxilios en un formulario o reporte web de primeros auxilios. Dicha información se recopila en línea (internet) en la base de datos transaccional. El segundo y tercer nivel de atención, los profesionales asistenciales y servicios de laboratorio, farmacia, diagnóstico de imágenes y las clínicas externas utilizan el sistema de historias clínicas electrónicas para registrar sus atenciones a través de computadoras conectadas a internet. En este sistema no sólo se recopilan datos estadísticos de su lesión o enfermedad, sino toda la información clínica del paciente se almacena en la base de datos. El efecto es que toda la información de los profesionales asistenciales se almacena en una única base de datos. A través de procedimientos ETL (Extract, Transform, Load) se extrae la información relevante que deba ser analizada. Esta información se almacena en otra base de datos llamada DataWarehouse mediante cubos OLAP.

La información así dispuesta puede ser consumida por los usuarios autorizados, mediante una plataforma web que actúa como capa de presentación. La información se muestra a través de gráficos interactivos, permitiendo al usuario realizar el análisis deseado, con data actualizada en el momento deseado (más detalles en Figura 2). Sobre la base del análisis de incidentes, por ejemplo, se ha podido mostrar que en el caso de Lima 2019, en contra de lo esperado, las atenciones asociadas a patología oral, específicamente, estaban asociadas al tratamiento de lesiones crónicas, lo que da pie a la implementación de sistemas más integrales de atención en salud para próximos juegos multideportivos
(Opazo-García et al., 2021). Puntualmente, a raíz de esta experiencia, se ha venido trabajando una aproximación al deportista desde una posición integral que combina la teoría de los determinantes sociales de la salud con la teoría sociobiológica informacional de la persona, resultando un enfoque predictivo, que empieza a revelar la necesidad de contemplar al deportista, más allá de un sujeto que rebosa bienestar, en un sujeto en condición de riesgo (justamente por la sobreexigencia que implica toda competencia).

\section{El atleta como sujeto de medición}

El deporte constituye una fuente de renovación, de efectos positivos para la salud. Sin embargo, en el caso del deporte asociado a eventos deportivos, a competencias, esta positividad del deporte para con la salud se ve alterada. Así, evidencia sistemática reciente ha revelado que los niveles de estrés que viven los deportistas, sobre todo en el tiempo que rondan su participación en competencias deportivas, se traducen en estados inmunitarios afectados, en tanto que disminuidos, y que facilitan el desarrollo de una serie de comorbilidades que afectan sostenible, y hasta en el largo plazo, en el rendimiento deportivo del atleta, que se ve disminuido (Gallagher et al., 2018; Bramantoro et al., 2020).

Ciertamente, una explicación integral del deportista debería incluir tanto la explicación de los efectos sociales de las condiciones deportivas, la emergencia de los defectos, trastornos y enfermedades en las personas (atletas) y el impacto en el rendimiento o performance deportivo. Gallagher et al. (2018) revelan la necesidad de comprender al atleta como un trabajador de su cuerpo, y no solo con su cuerpo. Sin embargo, se precisa una explicación sociobiológica que integre desde las células hasta la actividad consciente de los deportistas a fin de dar cuenta de todo el rango de manifestaciones mórbidas potenciales en este caso. En la Figura 2 se resume una explicación de este tipo, que pone énfasis en la necesidad de considerar a los depor tistas participantes en eventos de competencia como una población particularmente en riesgo, sobre la cual contar con sistemas de seguimiento y monitoreo confiables se constituye en una necesidad ineludible. Para contribuir a la resolución de esta necesidad es que, el COI, propuso y promueve el SVLE.

\section{Conclusión}

Nuestros resultados sugieren un adecuado uso del SVLE en diversos eventos multideportivos en los cinco 
continentes. A la luz de la literatura publicada, sería aquí la primera vez que se muestra una revisión de la experiencia del SVLE del COI a lo largo de más de una década de uso de este instrumento. Se entrega evidencia útil con respecto a facilitar la apropiación del SVLE en los próximos eventos deportivos a realizarse (Cali 2021, Tokio 2022, Chile 2023, y demás). Creemos que la utilidad concreta de nuestros aportes se medirá por la agilización al momento de la implementación del SVLE en próximos eventos deportivos (nacionales e internacionales). Se debe resaltar que el compromiso institucional y desde el más alto nivel de decisión deportiva es un imperativo necesario para llevar a buen término la aplicación y uso del SVLE en un evento multideportivo.

Si bien se han establecido diversos sistemas de vigilancia a lo largo de la historia del deporte, el SVLE presenta ventajas específicas como datos estandarizados (calidad de los datos), asegurar anonimato y confidencialidad de los datos, además de garantizar un cumplimiento y levantamiento de información óptimos y plausibles.

El SVLE representa una fortaleza en el emergente campo de la generación de evidencia en torno a eventos multideportivos (orientados a servir a la toma de decisiones en la planificación y gestión), posicionándose como un método sistematizado, estructurado y verídico. En este tipo de contextos, el SVLE del COI representa una herramienta útil, sencilla y ágil para el monitoreo de incidencias sanitarias.

\section{Fuente de financiamiento}

Este estudio es autofinanciado.

\section{Conflictos de intereses}

Los autores declaran no tener ningún conflicto de interés.

\section{Referencias}

Al-Shaqsi, S., Al-Kashmiri, A., Al-Risi, A., \& Al-Mawali, S. (2012). Sports injuries and illnesses during the second Asian Beach Games. The British Journal of Sports Medicine, 46(11), 780-787. doi:10.1136/bjsports2011-090852

Alonso, J. M., Junge, A., Renstrom, P., Engebretsen, L., Mountjoy, M., \& Dvorak, J. (2009). Sports injury surveillance during the 2007 IAAF World Athletics Championships. The Clinical Journal of Sport
Medicine, 19(1), 26-32 doi:10.1097/ jsm.0b013e318191c8e7

Alonso, J. M., Tscholl, P. M., Engebretsen, L., Mountjoy, M., Dvorak, J., \& Junge, A. (2010). Occurrence of injuries and illnesses during the 2009 IAAF World Athletics Championships. The British Journal of Sports Medicine, 44(15), 1100-1105. doi:10.1136/ bjsm.2010.078030

Alonso, J.M., Edouard, P., Fischetto, G., Adams, B., Depiesse, F., \& Mountjoy, M. (2012). Determination of future prevention strategies in elite track and field: analysis of Daegu 2011 IAAF Championships injuries and illnesses surveillance. The British Journal of Sports Medicine, 46(7), 505-514. doi:10.1136/bjsports2012- 091008

Bramantoro, T., Hariyani, N., Setyowati. D., Purwanto, B., Zulfiana, A.A., \& Irmalia, W.R. The impact of oral health on physical fitness: A systematic review. Heliyon. 2020; 6(4):e03774. doi: 10.1016/ j.heliyon.2020.e03774.

Bere, T., Alonso, J. M., Wangensteen, A., Bakken, A., Eirale, C., Dijkstra, H. P., ... Popovic, N. (2015) Injury and illness surveillance during the 24th Men's Handball World Championship 2015 in Qatar. The British Journal of Sports Medicine. 49(17), 1151-1156. doi:10.1136/bjsports-2015-094972

Crego, R. (2003) Sports and Games of the 18th and 19th Centuries. Connecticut: Greenwood Publishing Group.

Crowther, N.B. (2007) Sport in Ancient Times. Praeger series on the ancient world. Connecticut: Greenwood Publishing Group.

Dvorak, J., Junge, A., Derman, W., \& Schwellnus, M. (2011). Injuries and illnesses of football players during the 2010 FIFAWorld Cup. The British Journal of Sports Medicine. 45(8), 626-630. doi:10.1136/ bjsm.2010.079905

Edouard, P., Depiesse, F., Branco, P., \& Alonso, J. M. (2014).Analyses of Helsinki 2012 European Athletics Championships Injury and Illness Surveillance to Discuss Elite Athletes Risk Factors. The Clinical Journal of Sport Medicine, 24(5): 409-415. doi:10.1097/jsm.0000000000000052

Edouard, P., Depiesse, F., Hertert, P., Branco, P., \& Alonso, J.-M. (2012). Injuries and illnesses during the 2011 Paris European Athletics Indoor Championships. The Scandinavian Journal of Medicine \& Science in Sports. 23(4), e213-e218. doi:10.1111/ sms. 12027

Ekegren, C. L., Gabbe, B. J., \& Finch, C.F. (2015). Sports Injury Surveillance Systems: A Review of Methods 
and Data Quality. Sports Medicine, 46(1), 49-65. doi: 10.1007/s40279-015-0410-Z.

Engebretsen, L., Soligard, T., Steffen, K., Alonso, J.M., Aubry, M., Budgett, R., ... Renström, P.A. (2013). Sports injuries and illnesses during the London Summer Olympic Games 2012. The British Journal of Sports Medicine. 47(7): 407-414. doi:10.1136/ bjsports-2013-092380.

Engebretsen, L., Steffen, K., Alonso, J. M., Aubry, M., Dvorak, J., Junge, A., ... Wilkinson, M. (2010). Sports injuries and illnesses during the Winter Olympic Games 2010. The British Journal of Sports Medicine. 44(11), 772-780. doi:10.1136/ bjsm.2010.076992

Gallagher, J., Ashley, P., Petrie, A., \& Needleman, I. Oral health and performance impacts in elite and professional athletes. Comm dent oral epidemiol. 2018; 46(6): 563-568. https://doi.org/10.1111/ cdoe. 12392.

González-Ravé, J. M., Juárez Santos-García, D., \& Yustres, A. I. (2017). Análisis del rendimiento de las pruebas de natación en los JJOO «Rio2016. Retos. 32, 256-259. https://doi.org/10.47197/ retos.v0i32.56460

Joric, C. (2016). Berlín, 1936. Anillos y esvásticas. Historias y vida, 581, 30-39

Junge, A., Dvorak, J., Graf-Baumann, T., \& Petersonet, L. (2004). Football Injuries during FIFA Tournaments and the Olympic Games, 1998-2001. The American Journal of Sports Medicine. 32(Suppl 1), S80-S90. doi: 10.1177/0363546503261245

Junge, A., Engebretsen, L., Mountjoy, M. L., Alonso, J. M., Renström, P. A. F. H., Aubry, M. J., et al. (2009). Sports Injuries during the Summer Olympic Games 2008. The American Journal of Sports Medicine, 37(11), 2165-2172. doi:10.1177/0363546509339357

Junge, A., Langevoort, G., Pipe, A., Peytavin, A., Wong, F., Mountjoy, M., ... Dvorak, J. (2006). Injuries in Team Sport Tournaments during the 2004 Olympic Games. The American Journal of Sports Medicine, 34(4), 565-576. doi: 10.1177/0363546505281807.

Letelier, A. \& Orizola, A. (2015). Lesiones y Enfermedades de los Deportistas Durante los I Juegos Deportivos Nacionales. Chile- 2013. Revista de la Asociación Argentina de Traumatología del Deporte, 22(1).

Moher, D., Liberati, A., Tetzlaff, J. \& Altman D. G. (2009). The PRISMA Group Preferred Reporting Items for Systematic Reviews and Meta-Analyses: The PRISMA Statement. PLoS Medicine, 6(7), e1000097. https://doi.org/10.1371/ journal.pmed.1000097
Mountjoy, M., Junge, A., Alonso, J. M., Engebretsen, L., Dragan, I., Gerrard, D., ... Dvorak, J. (2010). Sports injuries and illnesses in the 2009 FINA World Championships (Aquatics). The British Journal of Sports Medicine, 44(7), 522-527. doi:10.1136/ bjsm.2010.071720

Muaidi, Q. I. (2019). Saudi Professional League: A Prospective Study of the Injuries and Illnesses Sustained by Professional Soccer Players During the 2015-2016 Season. The Asian Journal of Sports Medicine, 10(1), 1-8. doi: 10.5812/asjsm.79930.

Opazo-García, C., Moya-Salazar, J., Chicoma-Flores, K., \& Contreras-Pulache, H. (2021). Oral health problems in High-Performance Athletes at 2019 Pan American Games in Lima:A descriptive study. British Dental Journal Open. 7, 21. doi:10.1038/s41405-02100078-1

Ruedl, G., Schobersberger, W., Pocecco, E., Blank, C., Engebretsen, L., Soligard, T., ... Pocecco, E. (2012) Sport injuries and illnesses during the first Winter Youth Olympic Games 2012 in Innsbruck, Austria. The British Journal of Sports Medicine. 46(15): 10301037. doi:10.1136/bjsports-2012-091534

Sanz VA. (2000) La organización de grandes eventosdeportivos. Arbor, 151 (650), 265-287.

Soligard, T., Palmet, D., Steffen, K., Dias, L. A., Grant M-E., Kim, D.S., ... Engebretsen, L. (2019). Sports injury and illness incidence in the PyeongChang 2018 OlympicWinter Games: a prospective study of 2914 athletes from 92 countries. The British Journal of Sports Medicine, 53(17), 1085-1092. doi:10.1136/bjsports2018-100236.

Soligard, T., Steffen, K., Palmer-Green, D., Aubry, M., Grant, M.E., Meeuwisse, W., ... Engebretsen, L. (2015). Sports injuries and illnesses in the Sochi 2014 Olympic Winter Games. The British Journal of Sports Medicine, 49(7), 441-447. doi:10.1136/bjsports-2014094538

Soligard, T., Steffen, K., Palmer, D., Alonso, J. M., Bahr, R., Lopes, A. D., ... Engebretsen, L. (2017). Sports injury and illness incidence in the Rio de Janeiro 2016 Olympic summer Games: A prospective study of 11274 athletes from 207 countries. The British Journal of Sports Medicine, 51(17), 1265-1271. doi:10.1136/bjsports- 2017-097956.

Yoon, J.; Bae, M.; Kang, H. \& Kim, T. (2018). Descriptive epidemiology of sports injury and illness during the Rio 2016 Olympic Games: A prospective cohort study for Korean team. The International Journal of Sports Science \& Coaching, 13(6), 939-946. doi:10.1177/1747954118768686 